\title{
P-Glycoprotein-Mediated Drug Interactions in Pregnancy and Changes in the Risk of Congenital Anomalies: A Case-Reference Study
}

\author{
Aizati N. A. Daud ${ }^{1} \cdot$ Jorieke E. H. Bergman ${ }^{2} \cdot$ Marian K. Bakker $^{2} \cdot$ \\ Hao Wang ${ }^{3}$ - Wilhelmina S. Kerstjens-Frederikse ${ }^{2}$ - Hermien E. K. de Walle ${ }^{2}$. \\ Henk Groen ${ }^{2}$ Jens H. J. Bos ${ }^{3}$ Eelko Hak ${ }^{3} \cdot$ Bob Wilfert $^{1,4}$
}

Published online: 29 May 2015

(c) The Author(s) 2015. This article is published with open access at Springerlink.com

\begin{abstract}
Introduction Drug use in pregnancy is very common but may cause harm to the fetus. The teratogenic effect of a drug is partly dependent on the drug level in the fetal circulation, which is associated with the transport across the placenta. Many drugs are substrates of P-glycoprotein (P$\mathrm{gp})$, an efflux transporter that acts as a protective barrier for the fetus. We aim to identify whether drug interactions associated with P-gp promote any changes in fetal drug exposure, as measured by the risk of having children with congenital anomalies.

Methods In this study, cases $(N=4634)$ were mothers of children with congenital anomalies registered in the EUROCAT Northern Netherlands registry, and the reference population were mothers of children $(N=25,126)$ from a drug prescription database (IADB.nl).
\end{abstract}

Electronic supplementary material The online version of this article (doi:10.1007/s40264-015-0299-3) contains supplementary material, which is available to authorized users.

Aizati N. A. Daud

n.a.a.daud@rug.nl

1 Unit of Pharmacotherapy and Pharmaceutical Care, Department of Pharmacy, University of Groningen, Groningen, The Netherlands

2 Department of Genetics, University of Groningen, University Medical Center Groningen, Groningen, The Netherlands

3 Unit of Pharmacoepidemiology and Pharmacoeconomics, Department of Pharmacy, University of Groningen, Groningen, The Netherlands

4 Department of Clinical Pharmacy and Pharmacology, University of Groningen, University Medical Center Groningen, Groningen, The Netherlands
Results Drugs that are associated with P-gp transport were commonly used in pregnancy in cases $(10 \%)$ and population $(12 \%)$. Several drug classes, which are substrates for P-gp, were shown to have a higher user rate in mothers of cases with specific anomalies. The use of this subset of drugs in combination with other P-gp substrates increased the risk for specific anomalies (odds ratio [OR] $4.17,95 \%$ CI 1.75-9.91), and the addition of inhibitors further increased the risk (OR 13.03, $95 \%$ CI 3.37-50.42). The same pattern of risk increment was observed when the drugs were analyzed separately according to substrate specificity.

Conclusions The use of drugs associated with $\mathrm{P}$-gp transport was common during pregnancy. For several drug classes associated with specific anomalies, P-gp-mediated drug interactions are associated with an increased risk for those specific anomalies.

\section{Key Points}

Several drugs used in the first trimester of pregnancy are transported by P-glycoprotein (P-gp), an efflux transporter expressed in the placenta.

Some of these drugs were shown to have associations with specific types of anomalies. Drug interactions mediated by P-gp increase the risk of specific anomalies of these drugs compared with their use alone.

The pattern of risk increment following drug interactions suggests the role of P-gp in limiting fetal drug exposure. 


\section{Introduction}

Drug use in pregnancy is inevitable in some conditions, particularly in chronic diseases such as diabetes, depression and epilepsy. Drugs are also often prescribed to alleviate the symptoms associated with pregnancy or to fight infections [1]. In fact, between 27 and $93 \%$ of pregnant women in several developed countries were prescribed drugs, excluding vitamins and minerals, during pregnancy [2]. Moreover, the prescription rate during the first trimester is approximately $40 \%$, and nearly $3 \%$ of the pregnant women were prescribed drugs suspected to be teratogenic to the fetus $[1,3]$.

The risk of drug teratogenicity is dependent on the degree of fetal exposure, which is regulated partly by the transporter proteins in the placenta [4]. One of the most abundant transporter proteins is P-glycoprotein (P-gp), an efflux transporter expressed in the maternal-facing surface of the placental tissue $[5,6]$. P-gp acts as a barrier to preventing some potentially harmful drugs from entering the fetal circulation, and its function can be modulated by inhibitors and inducers $[5,7]$. Many drugs are known to be substrates of P-gp, some of which may also inhibit or induce this transporter. The use of P-gp inhibitors or inducers in combination with a substrate, referred to as 'P-gp-mediated drug interactions', may affect the P-gp barrier function in the placenta and subsequently fetal exposure to this substrate. For substrates that are teratogenic, the changes in transport and thereby of fetal exposure may be reflected in a changed risk of fetal congenital anomalies. Despite the importance of drug interactions in the placenta for fetal outcome, far too little attention has been given to this issue.

P-gp-mediated drug interactions have been previously explored in in vitro and in vivo studies [8, 9]. P-gp inhibitors increase the placental transfer of P-gp substrates in placental samples of late pregnancy [10]; however, the level of P-gp expression changes during pregnancy and the effect on fetal drug exposure during the early stage of pregnancy is not really known. There is still no established method to pursue this, particularly because of the difficulty in obtaining placental samples in early pregnancy.

The aim of this study was to explore the role of P-gp on fetal drug exposure in the first trimester of pregnancy. Applying a case-reference study design, we used the outcome of congenital anomalies as a proxy for fetal drug exposure. The first objective was to describe the pattern of use of drugs associated with P-gp transport during the first trimester of pregnancy, while the second objective was to determine the effect of P-gp-mediated drug interactions on the risk of congenital anomalies in exposed children.

\section{Methods}

\subsection{Case Selection}

Cases were selected among the mothers of children registered by EUROCAT Northern Netherlands (NNL), a population-based registry covering the northern provinces of The Netherlands (Groningen, Friesland and Drenthe). The registry includes fetuses or children, up to the age of 10 years, with congenital anomalies diagnosed before or after birth. All mothers of the children in this study gave consent to register their child in EUROCAT. EUROCAT records detailed information on the sociodemographic and lifestyle characteristics of the mothers, obtained through questionnaires. With the consent of the mothers, the information on drug use from 3 months before conception until delivery was collected from the pharmacy records and was later verified by telephone interviews. Among eligible children born in the study period (1997-2013), 81.6\% of parents gave consent to register their child in EUROCAT, and $80.2 \%$ returned the questionnaire and gave consent to retrieve their pharmacy data (personal communication). The actual use of both prescribed drugs, as well as overthe-counter (OTC) drugs, was completely registered, including the prescription status of any OTCs used (whether they were prescribed or not). Other details on drug use included, among others, the Anatomical Therapeutic Chemical (ATC) codes and the period of use during pregnancy.

The congenital anomalies were classified based on the EUROCAT Subgroup of Congenital Anomalies [11] and the International Classification of Diseases (ICD) coding system, ninth revision (ICD9) until 2001 and tenth revision (ICD10) from 2002 onwards. Only major anomalies were included in this study. The EUROCAT Subgroups of Congenital Anomalies (ICD9, ICD10) were anomalies of the nervous system (740-742, Q00-Q07), eye, ear, face and neck (743-744, Q10-Q18), heart (745, 746, 7470-7474, Q20-Q26), respiratory (748, Q30-Q34), orofacial clefts (7490-7492, Q35-Q37), digestive system (750-751, 7566, Q38-Q45, Q790), urinary (753, 75,672, 75,261, Q60-Q64, Q794), and genital (7520-7524, 75,260, 75,262, 7527-7529, Q50-Q52, Q54-Q56). Two subgroups of anomalies were classified according to the ICD10, i.e. (ICD9, ICD10) anomalies of the musculoskeletal system $(754,7566-7567$, Q65-Q66, Q790-Q795) and limb (755, Q69-Q74).

A total of 6934 children with congenital anomalies were born between 1 January 1997 and 31 December 2013. Overall, 885 children with genetic and chromosomal abnormalities were excluded because these anomalies were not likely to be related to drug use. We selected 5268 of the cases in which mothers had a history of drug use during 
pregnancy to match the reference population from a prescription database. Mothers with a previous history of children with anomalies were excluded in order to avoid selection bias in drug selection and prescribing, which led to recruitment of 4634 cases.

\subsection{Reference Population Sampling}

Reference data were obtained from the IADB.nl, a longitudinal, population-based prescription database in The Netherlands. The data were collected from 55 community pharmacies, including Groningen, Friesland and Drenthe, and covered an estimated population of 600,000 people. The prescription rates in this population have been found to be representative for the whole population in The Netherlands in comparison with data from insurance companies throughout the whole country [12]. Pharmacies periodically update the data of all delivered drugs for all patients, as prescribed by both general practitioners and specialists in the outpatient clinics. Data include the name of the prescribed drug, date of dispensing, quantity dispensed, dose regimen, prescribing physician and ATC codes [12].

A pregnancy database, Pregnancy IADB, was obtained from a large mother-child subset extracted from IADB.nl. All live-born children born between 1997 and 2013 were selected from this database. For each child, the female person aged 15-50 years older than the child and registered to the same address code is considered to be the mother. If there is another female person in that age range and with the same address code, the child will be excluded. Approximately $65 \%$ of children in the main IADB.nl database are linked to their mother, and validation of the identified mothers showed $99 \%$ accuracy [13].

The gestational period was determined from the theoretical conception date by subtracting the date of birth of the children with 273 days (gestational period of 9 months). Since the gestation period for twin and triplet pregnancies is likely to be shorter than singleton pregnancies, twin and triplet pregnancies were excluded. These pregnancies were identified based on the number of children with the same date of birth who were linked to the same mother. If more than one child was linked, the mother was excluded.

All registered pregnancies in the Pregnancy IADB, regardless of the number of pregnancies per mother, were the source population $(N=38,129)$. We then selected only the first registered pregnancy for each mother as the reference population $(N=25,126)$. The first known pregnancy for each mother was selected to overcome misclassification bias in maternal drug use since drug selection may be influenced by the previous pregnancy outcome. Ethical approval was not necessary for cases and population sampling since only anonymous data were used.

\subsection{Exposure Variable}

The user rates of drugs associated with P-gp transport during pregnancy were described in the source population to give a general overview of the prevalence at the population level. The P-gp drugs were identified through a literature review and classified according to substrate affinity, consisting of P-gp substrate, substrate/inhibitor, substrate/inducer, inhibitor, inducer, inhibitor/inducer and substrate/inhibitor/ inducer [5, 14-16]. This classification was determined by the results from in vivo studies and if not available, in vitro studies. More details on the list of all identified drugs are available in electronic supplementary Table 1. Drugs that are substrates/inducers are substrates when used alone but act as inducers to P-gp in the presence of another substrate. The same applies for substrates/inhibitors, which may inhibit the transport of another P-gp substrate when both are used concurrently. Inhibitors/inducers and substrates/inhibitors/inducers may become either one of these classes depending on substrate selectivity to P-gp in comparison with the other interacting drug.

The user rates include OTC drugs. The Dutch OTC drug list was obtained from the Medicines Evaluation Board of The Netherlands. The period of exposure was restricted to the period of organogenesis, i.e. the first trimester. Some drugs that are dispensed before the conception date may also be continually used during the first trimester, therefore we also included the preconception period. Children were considered to be exposed to the drugs if the period of the drugs dispensed/used was within 90 days before conception and the first 90 days of pregnancy.

To determine the effect of P-gp-mediated drug interactions on the risk of congenital anomalies, only drugs that showed associations with teratogenicity were selected. To find potential teratogenic drugs, we first grouped the drugs into drug classes according to their pharmacological action. Second, we calculated the user rates of these drug classes for all EUROCAT Subgroups of Congenital Anomalies. Drug classes with significantly higher user rates in specific subgroups of congenital anomalies were selected as 'drugs with associations'.

Information on the use of OTCs is not complete in the Pregnancy IADB; therefore, for a better comparison with cases, the use of OTC drugs was disregarded in this analysis. Throughout the study period, four drugs underwent changes in prescription regulation in The Netherlands (OTC to prescription drug, and vice versa), i.e. ranitidine, domperidone, omeprazole and pantoprazole. We included the exposure of these drugs among the case group, only when they were prescribed, so that the exposure status was comparable to the reference population.

Exposure was defined as mothers presented with P-gpmediated drug interaction patterns, which included the combination of drugs transported by P-gp with another substrate and/or inducer and/or inhibitor. 


\subsection{Statistical Analysis}

The analysis strategy for drug interaction patterns was conducted based on a priori hypothesis in which the distribution of types of medication used would differ between cases and the reference population, with an increased use in cases indicating a possible interaction. We also stratified the drug interaction analysis into substrate specificity (substrates, substrates/inhibitors) to observe whether the risk on anomalies was different for each group of drugs.

The Chi-square test was used in the comparison between the number of users in cases of specific anomalies and other anomalies. The number of cases of specific anomalies was then compared with the number of the reference population presented with the same pattern of P-gp-mediated drug interactions by calculating the odds ratio (OR) and $95 \%$ confidence interval (CI). An OR of more than 1 indicated an increased risk for congenital anomalies with certain interaction patterns. The results were considered as statistically significant at $p<0.05$. Since some drugs are classified as 'P-gp substrate' and some as 'P-gp substrate/ inhibitor', the analysis was conducted separately for each group. Analyses were performed using PASW Statistics, version 22 (IBM Corporation, Armonk, NY, USA).

\section{Results}

Characteristics of the children born to case mothers are presented in Table 1. The majority of children were live born, and the most common types of anomalies were the heart and musculoskeletal anomalies, which were each present in more than $20 \%$ of the children.

Maternal age at delivery was comparable between cases, source and reference populations, i.e. 30.3 years for case mothers, 30.0 years for mothers from the source population and 29.4 years for mothers from the reference population. A lower number of children were born in the last 5 years of the study period (2009-2013) in the source population $(18 \%)$ and reference population (15\%) compared with cases $(22 \%)$ because children were only registered in the IADB.nl once they received any prescription drug.

\subsection{User Rates of Drugs Associated with P- Glycoprotein (P-gp) During Pregnancy}

The use of drugs associated with P-gp transport is fairly common during pregnancy. Of $105 \mathrm{P}$-gp substrates identified in the literature, including OTC drugs, 44 were used by at least one case mother, while 66 were prescribed to at least one mother in the source population. The list of drugs and number of users among the cases and the reference population is available in the electronic supplementary
Table 1 Characteristics of children born to case mothers $(N=4634)$

\begin{tabular}{|c|c|c|}
\hline Characteristics & $N$ & $\%$ \\
\hline \multicolumn{3}{|l|}{ Sex } \\
\hline Boy & 2617 & 56.5 \\
\hline Girl & 2011 & 43.4 \\
\hline Missing & 6 & 0.1 \\
\hline \multicolumn{3}{|l|}{ Type of birth } \\
\hline Live birth & 4361 & 94.1 \\
\hline Termination of pregnancy & 184 & 4.0 \\
\hline Stillbirth & 58 & 1.3 \\
\hline Miscarriage (>24 weeks) & 31 & 0.7 \\
\hline \multicolumn{3}{|l|}{ Types of anomalies $^{\mathrm{a}}$} \\
\hline Heart & 1244 & 26.8 \\
\hline Musculoskeletal & 1054 & 22.7 \\
\hline Digestive system & 594 & 12.8 \\
\hline Urinary & 507 & 10.9 \\
\hline Orofacial clefts & 436 & 9.4 \\
\hline Genital & 406 & 8.8 \\
\hline Nervous system & 347 & 7.5 \\
\hline Limb & 342 & 7.4 \\
\hline Eye, ear, face and neck & 152 & 3.3 \\
\hline Respiratory & 84 & 1.8 \\
\hline
\end{tabular}

${ }^{\text {a }}$ Percentages do not add up to 100 as cases with multiple anomalies were counted more than once

Table 1. Furthermore, one or more of these drugs were used by $17.7 \%(n=820)$ of case mothers and $14.5 \%$ ( $n=5543)$ of mothers in the source population, suggesting that drugs associated with P-gp transport are widely used, even in pregnancy. The Pregnancy IADB also records the OTCs but only when the drugs are prescribed by physicians; therefore, the number of users in the source population is likely to be underestimated.

Among case mothers, $10.4 \%(n=481)$ had used at least one drug associated with P-gp transport, and $12.0 \%$ $(n=3022)$ of mothers in the reference population were prescribed these drugs. The user rates and prescription rates of these drugs, according to substrate specificity, are shown in Fig. 1. Doxycycline, a P-gp substrate, was the most commonly used or prescribed drug (case mothers, $n=102$ [2.2\%]; reference mothers, $n=595$ [2.4\%]), followed by omeprazole, a P-gp substrate/inhibitor (case mothers, $n=41$ [0.9\%]; reference mothers, $n=294$ [1.2\%]). For drug groups indicated for chronic diseases, the user rate of selective serotonin reuptake inhibitors (SSRIs) was the highest [case mothers, $n=86(1.9 \%)$; reference mothers, $n=607(2.4 \%)]$ compared with other antidepressants, antipsychotics and antiepileptics.

In the selection of drugs with associations, most OTCs were excluded, as explained in the Methods section. Among cases, 13 drugs were identified that were used in a higher percentage in cases with specific anomalies 


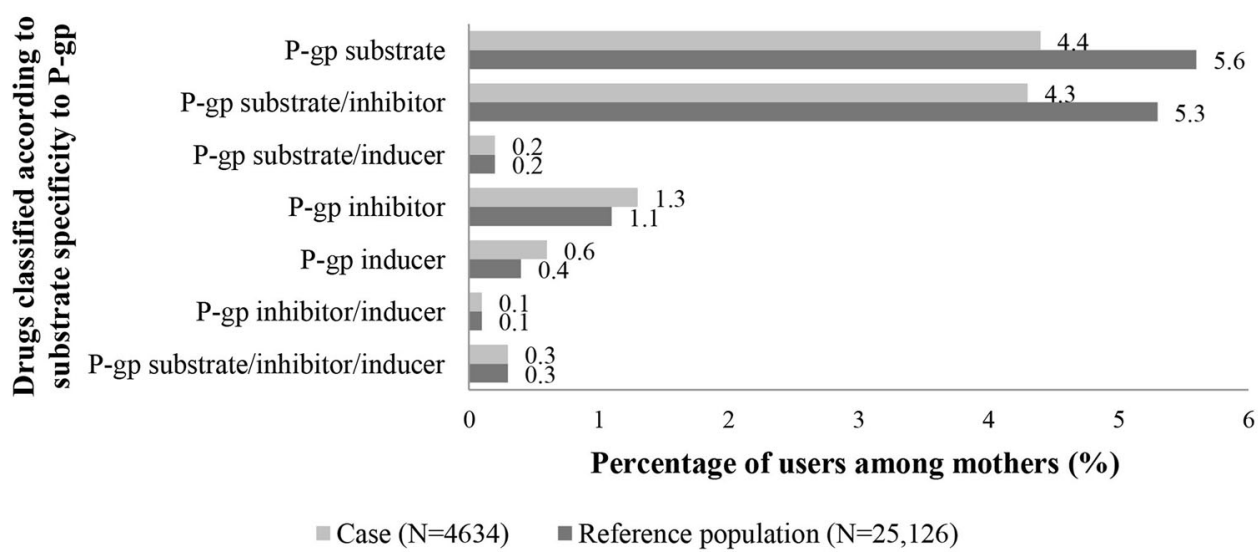

Fig. 1 User rates (in case mothers) and prescription rates (in mothers of the reference population) of drugs associated with P-gp transport. One mother may be counted more than once. The list of all drugs associated with P-gp transport is available in Table 1 of the electronic supplementary material. The classifications of drugs according to P-gp substrate specificity and drugs with at least one user in both cases and reference population are as follows: P-gp substrate (cimetidine, ranitidine, domperidone, propranolol, betamethasone, hydrocortisone, triamcinolone, doxycycline, tetracycline, sumatriptan, lamotrigine, levetiracetam, risperidone, clomipramine, nortriptyline, citalopram, venlafaxine, fexofenadine); P-gp substrate/inhibitor (omeprazole, pantoprazole, simvastatin, atorvastatin, clarithromycin, azithromycin, ketoconazole, itraconazole, cyclosporine, haloperidol, quetiapine, amitriptyline, fluoxetine, paroxetine, sertraline, fluvoxamine, terfenadine); P-gp substrate/inducer (dexamethasone, morphine, phenytoin, carbamazepine); P-gp inhibitor (progesterone, duloxetine, mefloquine); P-gp inducer (insulin); P-gp inhibitor/inducer (bromocriptine, midazolam); P-gp substrate/ inhibitor/inducer (erythromycin, diltiazem). $P$ - $g p$ P-glycoprotein

compared with cases with other anomalies (Table 2). Cimetidine and ranitidine $\left(\mathrm{H}_{2}\right.$-receptor antagonists) were shown to have an association with heart anomalies ( $p=0.037$ ), omeprazole and pantoprazole (proton pump inhibitors [PPIs]) with genital anomalies $(p=0.046)$, morphine with respiratory anomalies $(p=0.018)$, and antipsychotics (haloperidol, quetiapine and risperidone) with musculoskeletal anomalies $(p=0.018)$. Drugs that were found to have associations with specific anomalies were grouped as 'drugs with associations' and were used in the risk estimation analysis for P-gp-mediated drug interactions in congenital anomalies. The association between SSRIs (paroxetine, sertraline, fluoxetine, fluvoxamine, citalopram) and nervous system anomalies nearly reached statistical significance $(p=0$. 054), but due to previous warnings of teratogenicity, this drug class was also classified as 'drugs with associations'.

Additional analysis on the risk of specific anomalies with the respective drug class was carried out by comparing the user rates in case mothers and mothers in the reference population. Increases in ORs were found for most of the drug classes with specific anomalies, but failed to reach statistical significance.

\subsection{P-gp-Mediated Drug Interactions and the Risk of Congenital Anomalies}

The risk of congenital anomalies was found to be affected by P-gp-mediated drug interactions (Fig. 2). For all P-gp substrates, including drugs without associations, we could not show an effect of the combination with any inhibitor or inducer on the risk for overall anomalies compared with the use of the substrate alone (OR 0.81, $95 \%$ CI 0.52-1.27, $p=0.4$ ). However, the risk for specific anomalies was significantly increased to nearly threefold in mothers using any of the 13 drugs with associations in combination with other substrates (OR 4.17, $95 \%$ CI 1.75-9.91, $p=0.003$ ). The risk was further augmented up to sixfold with the addition of inhibitors (OR 13.03, $95 \%$ CI 3.37-50.42, $p=0.003$ ). The risk estimations for individual drugs with associations are available in electronic supplementary Table 2 .

The same pattern of risk increment was observed when analyses were conducted separately for substrates and substrate/inhibitor groups within the drugs with associations. For substrates (cimetidine, ranitidine, risperidone, citalopram), herein referred to as 'drug', the use of 'drug + substrate(s)' significantly increased the risk of specific anomalies compared with its use alone (OR 7.64, $95 \%$ CI 1.61-36.33, $p=0.023)$. The combination of 'drug + substrate(s) + inhibitor(s)' showed a slight increase in risk but failed to reach statistical significance, probably because of the low number of exposed cases. For substrates/inhibitors (omeprazole, pantoprazole, haloperidol, quetiapine, fluoxetine, paroxetine, sertraline, fluvoxamine), the combination of 'drug + substrate(s)' significantly increased the risk of specific anomalies by approximately threefold (OR 3.21, $95 \%$ CI 1.12-9.23, $p=0.04$ ). The 
Table 2 Number of users of drug/drug classes associated with specific congenital anomalies, and the risk determination of these anomalies from the comparison with the reference population

\begin{tabular}{|c|c|c|c|c|c|c|c|}
\hline \multirow{2}{*}{$\begin{array}{l}\text { Drug/drug } \\
\text { classes }\end{array}$} & \multirow{2}{*}{$\begin{array}{l}\text { Types of specific } \\
\text { anomalies }\end{array}$} & \multicolumn{2}{|c|}{ Number of case users $(\%)$} & \multirow[t]{2}{*}{$p$ value } & \multirow{2}{*}{$\begin{array}{l}\text { Number of users } \\
\text { in reference } \\
\text { population } \\
{[N=25,126]} \\
(\%)\end{array}$} & \multirow{2}{*}{$\begin{array}{l}\text { OR } \\
(95 \% \mathrm{CI})\end{array}$} & \multirow[t]{2}{*}{$p$ value } \\
\hline & & $\begin{array}{l}\text { Within } \\
\text { specific } \\
\text { anomalies }\end{array}$ & $\begin{array}{l}\text { Within all other } \\
\text { anomalies } \\
{\left[N=4634-n_{\mathrm{s}}\right]}\end{array}$ & & & & \\
\hline $\begin{array}{l}\mathrm{H}_{2} \text {-receptor antagonist } \\
\text { (cimetidine, ranitidine) }\end{array}$ & Heart $\left(n_{\mathrm{s}}=244\right)$ & $5(0.4)$ & $3(0.1)$ & $0.037^{\mathrm{a}}$ & $161(0.64)$ & $0.63(0.26-1.53)$ & 0.3 \\
\hline $\begin{array}{l}\text { Proton pump inhibitors } \\
\text { (omeprazole, } \\
\text { pantoprazole) }\end{array}$ & $\begin{array}{l}\text { Genital } \\
\qquad\left(n_{\mathrm{s}}=406\right)\end{array}$ & $9(2.2)$ & $52(1.2)$ & 0.046 & $327(1.3)$ & $1.72(0.88-3.36)$ & 0.11 \\
\hline Morphine & $\begin{array}{r}\text { Respiratory } \\
\left(n_{\mathrm{s}}=84\right)\end{array}$ & $1(1.2)$ & $7(0.2)$ & $0.018^{\mathrm{a}}$ & $3(0.01)$ & $100.9(10.39-979.94)$ & $0.13^{\mathrm{a}}$ \\
\hline $\begin{array}{l}\text { Antipsychotics } \\
\text { (haloperidol, quetiapine, } \\
\text { risperidone) }\end{array}$ & $\begin{array}{l}\text { Musculoskeletal } \\
\quad\left(n_{\mathrm{s}}=1054\right)\end{array}$ & $5(0.5)$ & $3(0.1)$ & $0.018^{\mathrm{a}}$ & $57(0.23)$ & $2.1(0.84-5.24)$ & 0.1 \\
\hline $\begin{array}{l}\text { SSRIs (fluoxetine, } \\
\text { citalopram, paroxetine, } \\
\text { sertraline, fluvoxamine) }\end{array}$ & $\begin{array}{l}\text { Nervous system } \\
\quad\left(n_{\mathrm{s}}=347\right)\end{array}$ & $11(3.2)$ & $74(1.7)$ & $0.054^{\mathrm{b}}$ & $576(2.3)$ & $1.4(0.76-2.56)$ & 0.28 \\
\hline
\end{tabular}

$C I$ confidence interval, $n_{s}$ number of cases of respective type of anomalies, OR odds ratio, SSRIs selective serotonin reuptake inhibitors

${ }^{a}$ Fisher's exact test

b Although not significant, SSRIs will be included in further analysis due to previous warnings of teratogenicity

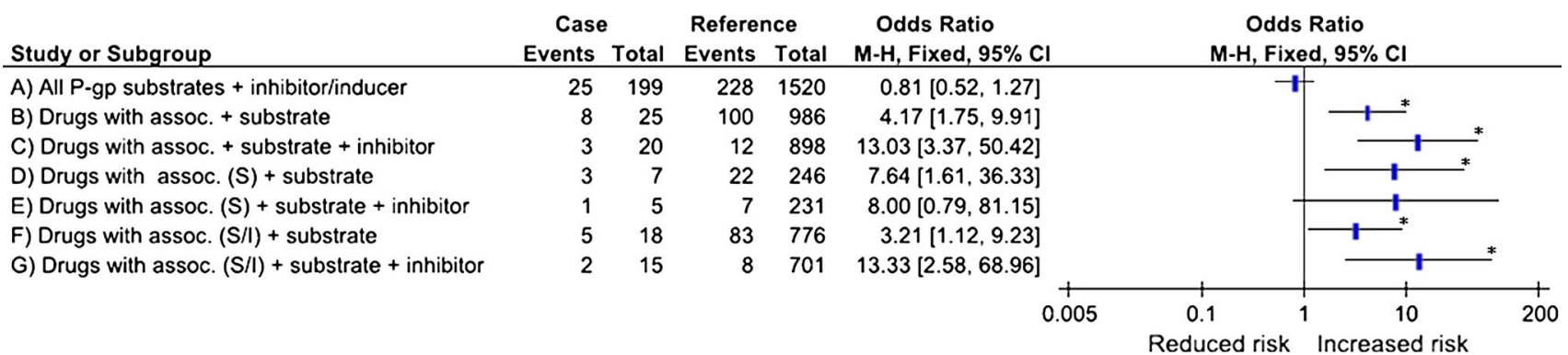

Fig. 2 The risk estimation of overall and specific anomalies with several patterns of P-gp-mediated drug interactions. The reference for the odds ratio calculation is the use of drug substrates alone for each subgroup. $a$ Showed no increased risk of overall anomalies associated with drug interactions in $41 \mathrm{P}$-gp substrates, $p=0.4$ (see Fig. 1 for the list of drugs); $b, c$ showed the risk changes for specific anomalies (heart, genital, respiratory, musculoskeletal and nervous system) in 13 drugs previously associated with these anomalies (cimetidine, ranitidine, omeprazole, pantoprazole, morphine, haloperidol, risperidone, quetiapine, citalopram, fluoxetine, paroxetine, sertraline, fluvoxamine), both with $p=0.003$; $d, e$ showed the risk changes for specific anomalies (heart, musculoskeletal and nervous system) in P-gp substrates previously associated with these anomalies (cimetidine, ranitidine, risperidone, citalopram), $p=0.023$ and 0.16 , respectively; $f, g$ showed the risk changes for specific anomalies (genital, musculoskeletal and nervous system) in P-gp substrates/inhibitors previously associated with these anomalies (omeprazole, pantoprazole, haloperidol, quetiapine, fluoxetine, paroxetine, sertraline, fluvoxamine), $p=0.04$ and 0.017 , respectively. assoc. associations, $C I$ confidence interval, $M$ - $H$ Mantel-Haenszel, $P$-gp P-glycoprotein, $S$ substrates, S/I substrates/inhibitors. Asterisk indicates $p<0.05$ (Fisher's exact test)

addition of inhibitor(s) further increased the risk up to tenfold, but with a large confidence interval (OR 13.33, $95 \%$ CI 2.58-68.96, $p=0.017$ ).

Morphine was the only substrate/inducer used in both cases and the reference population-one user among cases and three users in the reference population. These numbers impede further analysis of drug interactions for morphine. However, this drug was included in the analysis where all drugs with associations were combined.

\section{Discussion}

Our study was the first to describe the pattern of use of drugs associated with P-gp transport in the first trimester of pregnancy. We also found that P-gp-mediated drug interactions have an effect on the risk of congenital anomalies, as a proxy for fetal exposure. It has already been shown that these interactions affect drug pharmacokinetics and the pharmacodynamic effects of multiple drugs, mostly based 
on the interactions occurring in the small intestine, liver and kidney (see review by Akamine et al. [16]). Since P-gp is also substantially expressed in the placenta, the P-gp inhibition and induction is expected to play a role in determining fetal drug exposure. P-gp inhibition is expected to lead to a higher amount of drugs transferred into the fetal circulation, which can potentially harm the fetus. The pattern of risk increment between 'drug + substrate(s)' and 'drug + substrate(s) + inhibitor(s)' was the same for P-gp substrates and substrates/inhibitors, although we found a large CI due to a limited number of exposed mothers.

Several preclinical studies have demonstrated the role of P-gp inhibition in the drug transport mechanism in the placenta, using placental samples after delivery (approximately 37-42 weeks of gestation). In studies using the dually perfused human placental model, the maternal-tofetal transport of saquinavir, indinavir and lopinavir was shown to increase with P-gp inhibition [17, 18]. The transfer of paclitaxel, methadone and talinolol were also affected by the drug interactions mediated by P-gp [19-21]. In order to evaluate the role of P-gp-mediated drug interactions in the risk of congenital anomalies, it is important that the P-gp level in preclinical studies is comparable to that observed in the early stages of pregnancy. As the expression level of P-gp is known to decrease when pregnancy advances [22, 23], it may be expected that the results of these preclinical studies may not fully demonstrate the degree of P-gp inhibition and the effect on fetal drug exposure in the first trimester of pregnancy. The placental expression of P-gp itself is also subject to interindividual variation, in which the expression level may differ among mothers [19]. However, the correlation between expression level and activity of the placental P-gp also remains unclear, leading to the need for different methods in order to evaluate the net effect of placental drug transfer [19].

Apart from P-gp transport, there are other factors that may contribute to the net concentration of a drug in the placenta. These factors include changes in placental permeability which may readily alter maternal-to-fetal drug disposition [24]. Increased permeability of the placenta is caused by a reduction in placental thickness, as well as increased surface area and placental blood flow. Another factor is the drug interactions mediated by the metabolic enzymes, the cytochrome (CYP) 450. P-gp and CYP450 enzymes were shown to share a broad substrate specificity $[16,25]$. In this study, we assume that, at least from 2002, the clinically significant CYP-mediated drug interactions had already been avoided during prescribing and dispensing by the software-supported medications surveillance commonly used in The Netherlands [26]. Furthermore, the metabolic enzymes expressed in the placenta seemed to add minor contributions to fetal drug transfer [10]. The last factor was the genetic variability of the genes encoding for
P-gp and CYP450 relevant to the pharmacokinetics of each drug investigated. Genotype-dependent drug interactions can also cause variations in the drug's efficacy and toxicity profile [27, 28].

Since this study aims to explore the role of P-gp in fetal drug exposure using a technical approach, the list of drugs tested in this study was based on in vivo and in vitro studies with regard to their substrate specificity to P-gp transport. A drug identified in an in vitro study is not necessarily of clinical importance, and extrapolating from animal evidence to humans is not always simple. To improve the quality of results in future studies, it is suggested to focus on specific drug classes with documented clinical evidence on P-gp transport activity.

Although the proportion of drug transferred across the placenta is considered minimal, any change in the placental barrier mechanism may cause significant changes in fetal drug concentration. These changes can be detrimental to the fetus, especially if the drugs have a small therapeutic index, are teratogenic, or can potentially affect the normal physiological transport of nutrients to the fetus [29].

\subsection{Strengths and Limitations}

Our study was the first to report on the occurrence of P-gpmediated drug interactions in pregnant women and the effect on the fetus. Drug use and the period of use are welldocumented in both databases used in this study, and the cases and population sampling were carried out in the same time period. Moreover, the prescription records in the IADB.nl are virtually complete due to the high patientpharmacy commitment in The Netherlands [12].

In the determination of the 'drugs with associations', we did not find the previously reported associations between drug or drug groups with specific types of anomalies, for example antiepileptics and neural tube defects [30]. There are several reasons for this. First, our study was not set up to find these associations because we focused on P-gpmediated drug interactions and therefore we used the user rates from cases with all other anomalies within our case group as a reference group. It is a convenient method to quickly identify potentially harmful drugs and then continue with the next analysis to identify whether drug interactions may change the risk. In contrast, in most pharmacovigilance studies, the aim is to find a signal of teratogenicity of a drug, and the major malformation rates are usually calculated by using total birth as a denominator. Second, our study might be underpowered to detect previously reported associations.

One of our limitations was selection bias in the reference population since only children who had received prescription drugs were registered in the IADB.nl. In the IADB.nl, only $65 \%$ of children in the population could be 
linked to their mother. The most important reasons for missing the linkage were that the mother had a separate address registration number, the mother was registered with another pharmacy in the town, or the mother did not live at the same address [13]. We do not have information on the non-linked children; however, since linkage is based on birth date and address rather than health profiles, the linkage may have been random and is unlikely to have substantially influenced exposure and outcome. Although the Pregnancy IADB can only link a child to a mother if they had taken prescription drugs, it is well-known that approximately $80 \%$ of children, before the age of 2 years, have been prescribed at least one medication that was dispensed from the pharmacy [31]. We therefore assumed that the reference population was comparable to the unselected group.

There was also a potential classification bias in the exposure period of drugs in the reference population as the gestational period of the pregnancies in the reference population was based on estimation (assumption of 293 days of gestation). Furthermore, exposure definition differs between cases and the reference population, which is based on actual use and prescriptions, respectively. Actual exposure in the reference population is expected to be lower than observed, which may have led to an underestimation of the observed increased risk. IADB.nl only registered the live births, therefore we cannot include stillborn children and pregnancies that underwent miscarriage or termination. There is a possibility that the risk estimations for congenital anomalies in the results are overestimated if the exposure rate in these children/pregnancies are higher compared with live-born children. However, due to a low rate of stillbirths, miscarriages and terminations of pregnancy in the population-based statistics $(0.2-0.4 \%$ for stillbirths [32]), we expect the change to be very minimal. A similar assumption applies to children in the Pregnancy IADB who might also have congenital anomalies. The rate of congenital anomalies is approximately $3 \%$ of the total number of births, therefore these numbers are not expected to cause significant changes in risk estimation [33].

Confounding by indication was also assumed to have minor implications on the results. This type of confounding refers to those situations in which the indication for treatment acts as a confounder, in this case the disease influences the exposure to P-gp substrates and acts as a risk factor for congenital anomalies. In this study, the exposure variable consists of a broad range of drugs, each with specific indications. One of the known risk factors for congenital anomalies is maternal diabetes mellitus [34], and insulin is one of the drugs associated with P-gp transport; however, insulin is an inducer to this transporter, not a substrate, therefore it is not included in the analysis for the effect of P-gp inhibition on the risk of congenital anomalies.
There are also other confounding factors that we were not able to address, such as maternal smoking behavior, alcohol consumption and other risk factors for congenital anomalies. Therefore, our results warrant further epidemiological studies, preferably with a higher number of exposed cases and focusing on exposure to selected teratogenic drugs in specific congenital anomalies in combination with potent inhibitors or inducers.

\section{Conclusions}

The use of drugs associated with P-gp transport was common in pregnancy, and some mothers had to use more than one of these drugs. For several drug classes that were associated with specific anomalies, P-gp-mediated drug interactions were found to increase the risk for those anomalies. Apart from other factors that may affect fetal drug exposure, for these drug classes, P-gp plays a clinically relevant role in limiting drug exposure, as previously suggested in preclinical studies. The absolute risk of such interactions may be modest, but at the P-gp level it showed a significant increment in the risk of congenital anomalies in drugs suspected to be teratogenic. Moreover, this knowledge may assist drug individualization in pregnancy, especially in chronic diseases.

Acknowledgments The authors would like to acknowledge Linda de Jonge for her technical advice with the EUROCAT NNL database.

Compliance with ethical standards Funding EUROCAT NNL is funded by the Ministry of Health, Welfare and Sports, The Netherlands. The IADB.nl database is funded by the University of Groningen, Groningen, The Netherlands.

Conflict of interest Aizati Daud, Jorieke Bergman, Marian Bakker, Hao Wang, Wilhelmina Kerstjens-Frederikse, Hermien de Walle, Henk Groen, Jens Bos, Eelko Hak and Bob Wilffert have no conflicts of interest to declare that are directly relevant to the content of this study.

Open Access This article is distributed under the terms of the Creative Commons Attribution-NonCommercial 4.0 International License (http://creativecommons.org/licenses/by-nc/4.0/), which permits any noncommercial use, distribution, and reproduction in any medium, provided you give appropriate credit to the original author(s) and the source, provide a link to the Creative Commons license, and indicate if changes were made.

\section{References}

1. Bakker MK, Jentink J, Vroom F, Van Den Berg PB, De Walle HEK, De Jong-Van Den Berg LTW. Drug prescription patterns before, during and after pregnancy for chronic, occasional and pregnancy-related drugs in the Netherlands. BJOG. 2006;113:559-68. 
2. Daw JR, Hanley GE, Greyson DL, Morgan SG. Prescription drug use during pregnancy in developed countries: a systematic review. Pharmacoepidemiol Drug Saf. 2011;20:895-902.

3. Andrade SE, Gurwitz JH, Davis RL, Chan KA, Finkelstein JA, Fortman K, et al. Prescription drug use in pregnancy. Am J Obstet Gynecol. 2004;191:398-407.

4. Staud F, Cerveny L, Ceckova M. Pharmacotherapy in pregnancy: effect of $\mathrm{ABC}$ and SLC transporters on drug transport across the placenta and fetal drug exposure. J Drug Target. 2012;20:736-63.

5. Schinkel AH, Jonker JW. Mammalian drug efflux transporters of the ATP binding cassette (ABC) family: an overview. Adv Drug Deliv Rev. 2003;55:3-29.

6. Ceckova-Novotna M, Pavek P, Staud F. P-glycoprotein in the placenta: expression, localization, regulation and function. Reprod Toxicol. 2006;22:400-10.

7. Zhou SF. Structure, function and regulation of P-glycoprotein and its clinical relevance in drug disposition. Xenobiotica. 2008;38:802-32.

8. Balayssac D, Authier N, Cayre A, Coudore F. Does inhibition of P-glycoprotein lead to drug-drug interactions? Toxicol Lett. 2005;156:319-29.

9. Aszalos A. Drug-drug interactions affected by the transporter protein, P-glycoprotein (ABCB1, MDR1) I. Preclinical aspects. Drug Discov Today. 2007;12:833-7.

10. Rubinchik-Stern M, Eyal S. Drug interactions at the human placenta: what is the evidence? Front Pharmacol. 2012;3:1-7.

11. European Surveillance of Congenital Anomalies (EUROCAT). EUROCAT guide 1.3 and reference documents. Instructions for the registration and surveillance of congenital anomalies. 2005. Available at: http://www.eurocat-network.eu/content/EUROCAT -Guide-1.3.pdf. Accessed 27 Oct 2014.

12. Visser ST, Schuiling-Veninga CC, Bos JH, de Jong-van den Berg LTW, Postma MJ. The population-based prescription database IADB.nl: its development, usefulness in outcomes research and challenges. Expert Rev Pharmacoecon Outcomes Res. 2013;13:285-92.

13. Schirm E, Tobi H, de Jong-van den Berg LTW. Identifying parents in pharmacy data: a tool for the continuous monitoring of drug exposure to unborn children. $\mathrm{J}$ Clin Epidemiol. 2004;57:737-41.

14. Endres CJ, Hsiao P, Chung FS, Unadkat JD. The role of transporters in drug interactions. Eur J Pharm Sci. 2006;27:501-17.

15. Hodges LM, Markova SM, Chinn LW, Gow JM, Kroetz DL, Klein TE, et al. Very important pharmacogene summary: ABCB1 (MDR1, P-glycoprotein). Pharmacogenet Genomics. 2011;21:152-61.

16. Akamine Y, Yasui-Furukori N, Ieiri I, Uno T. Psychotropic drugdrug interactions involving P-glycoprotein. CNS Drugs. 2012;26:959-73.

17. Molsa M, Heikkinen T, Hakkola J, Hakala K, Wallerman O, Wadelius M, et al. Functional role of P-glycoprotein in the human blood-placental barrier. Clin Pharmacol Ther. 2005;78:123-31.

18. Ceccaldi P-F, Gavard L, Mandelbrot L, Rey E, Farinotti R, Treluyer J-M, et al. Functional role of p-glycoprotein and binding protein effect on the placental transfer of lopinavir/ritonavir in the ex vivo human perfusion model. Obstet Gynecol Int. 2009;2009:726593.

19. Nanovskaya T, Nekhayeva I, Karunaratne N, Audus K, Hankins GD, Ahmed MS. Role of P-glycoprotein in transplacental transfer of methadone. Biochem Pharmacol. 2005;69:1869-78.

20. May K, Minarikova V, Linnemann K, Zygmunt M, Kroemer HK, Fusch $\mathrm{C}$, et al. Role of the multidrug transporter proteins ABCB1 and $\mathrm{ABCC} 2$ in the diaplacental transport of talinolol in the term human placenta. Drug Metab Dispos. 2008;36:740-4.

21. Hemauer SJ, Patrikeeva SL, Nanovskaya TN, Hankins GD, Ahmed MS. Opiates inhibit paclitaxel uptake by P-glycoprotein in preparations of human placental inside-out vesicles. Biochem Pharmacol. 2009;78:1272-8.

22. Sun M, Kingdom J, Baczyk D, Lye SJ, Matthews SG, Gibb W. Expression of the multidrug resistance P-glycoprotein (ABCB1 glycoprotein) in the human placenta decreases with advancing gestation. Placenta. 2006;27:602-9.

23. Gil S, Saura R, Forestier F, Farinotti R. P-glycoprotein expression of the human placenta during pregnancy. Placenta. 2005;26:268-70.

24. Unadkat JD, Dahlin A, Vijay S. Placental drug transporters. Curr Drug Metab. 2004;5:125-31.

25. Kim RB. Drugs as P-glycoprotein substrates, inhibitors, and inducers. Drug Metab Rev. 2002;34:47-54.

26. Van Roon EN, Flikweert S, Le Comte M, Langendijk PNJ, Kwee-Zuiderwijk WJM, Smits P, et al. Clinical relevance of drug-drug interactions: a structured assessment procedure. Drug Saf. 2005;28:1131-9.

27. Muller F, Fromm MF. Transporter-mediated drug-drug interactions. Pharmacogenomics. 2011;12:1017-37.

28. Blumenfeld YJ, Reynolds-May MF, Altman RB, El-Sayed YY. Maternal-fetal and neonatal pharmacogenomics: a review of current literature. J Perinatol. 2010;30:571-9.

29. Prouillac C, Lecoeur S. The role of the placenta in fetal exposure to xenobiotics: importance of membrane transporters and human models for transfer studies. Drug Metab Dispos. 2010;38:1623-35.

30. Tomson T, Battino D. Teratogenic effects of antiepileptic drugs. Lancet Neurol. 2012;11:803-13.

31. Schirm E, van den Berg P, Gebben H, Sauer P, De Jong-van den Berg L. Drug use of children in the community assessed through pharmacy dispensing data. Br J Clin Pharmacol. 2000;50:473-8.

32. Cousens S, Blencowe H, Stanton C, Chou D, Ahmed S, Steinhardt L, et al. National, regional, and worldwide estimates of stillbirth rates in 2009 with trends since 1995: a systematic analysis. Lancet. 2011;377:1319-30.

33. European Surveillance of Congenital Anomalies (EUROCAT). EUROCAT prevalence data tables. 2015. Available at: http:// www.eurocat-network.eu/ACCESSPREVALENCEDATA/Preval enceTables. Accessed 12 Dec 2014.

34. Ornoy A, Reece EA, Pavlinkova G, Kappen C, Miller RK. Effect of maternal diabetes on the embryo, fetus, and children: congenital anomalies, genetic and epigenetic changes and developmental outcomes. Birth Defects Res C Embryo Today. 2015;105:53-72. 